\title{
Paradoxes in understanding the Efficiency Factors of Space Heating
}

\author{
Christian Brembilla (D) • Ronny Östin • \\ Mohsen Soleimani-Mohseni · Thomas Olofsson
}

Received: 1 December 2017 / Accepted: 24 May 2018 / Published online: 5 July 2018

(C) The Author(s) 2018

\begin{abstract}
Efficiency factors are here defined as the thermal energy performance indicators of the space heating. Until recently, the efficiency factors were assumed as one value for space heating located in any climate. This study addresses the problem of how the outdoor climate affects the efficiency factors of a space heating equipped with 1D model of hydronic floor heating. The findings show how the efficiency factors, computed with two numerical methods, are correlated with the solar radiation. This study highlights the paradoxes in understanding the results of efficiency factors analysis. This work suggests how to interpret and use the efficiency factors as a benchmark performance indicator.
\end{abstract}

Keywords Efficiency factors - Space heating . Accuracy 1D model of hydronic floor heating . Outdoor climate $\cdot$ Performance indicator $\cdot$ Solar radiation $\cdot$ Benchmark $\cdot$ Linear regression model

\section{Introduction}

Climate of different localities affected the efficiency factors of the space heating as stated in Brembilla

\footnotetext{
C. Brembilla $(\varangle) \cdot$ R. Östin · M. Soleimani-Mohseni ·

T. Olofsson

Department of Applied Physics and Electronics

(TFE), Umeå University, X Håken Gullessons väg,

20 Teknikhuset, SE-901 87 Umeå, Sweden

e-mail: christian.brembilla@gmail.com
}

et al. (2017); but, the reasons for these discrepancies were unclear. Other authors, see Goubran et al. (2017), stated that "...air curtain door generally increases in its efficiency in reducing whole building energy in colder climates." At first glance, the reported result appears as a paradox. Readers can conclude that air curtains have to be installed in colder climates because they can achieve higher efficiencies in reducing the building energy usage. It is crucial to understand why the outdoor climate affects the efficiency factors. This information increases the understanding of how to interpret this performance indicator dissolving the misleading information on this topic. In addition, it would be possible to forecast the efficiency factors by relating them with the outdoor climate conditions. As hypothesis, the efficiency factors vary according to the climate of each locality.

Several factors are related to the outdoor climate; but, some of them can have a minor contribution on the efficiency factors. For instance, the wind influences the external convective heat transfer coefficient of the building façade, see Shao et al. (2010), Loveday and Taki (1996). The cloudiness factor affects the heat transfer between the building outer surface and the sky dome without taking into account the relative position of the sun and the cloud as stated in Paulescu et al. (2011). Both wind and cloudiness factors are considered here negligible. Such factors produce a minor effect on the building energy use as shown in Emmel et al. (2007) for the wind, and as a result on the efficiency factors. Such hypothesis 
is further confirmed by the relatively low thermal transmittance of the building envelope (assumed here $0.15 \mathrm{Wm}^{-2} \mathrm{~K}^{-1}$ ). The latter parameter decreases the impact of these disturbances on the building energy use (and on the efficiency factors) as well as smoothing the influence of the sharp variations of outdoor temperature as shown in Asan (2006).

The efficiency factors aim to measure how "effectively" the thermal energy supplied to the floor is released into the room control volume. This method, investigated in Abdullah and Ali (2009), is here defined with the name common practice. A second method, studied in Olesen and De Carli (2011), intended to measure the system thermodynamic in/efficiencies of the space heating. Similar considerations on how to define the thermodynamic in/efficiencies were stated in Rosen (2002), Dinçer and Rosen (2010) for assessing exergy factors of thermal energy storage. The space heating is de facto a thermal energy storage because the heat is stored in the building thermal mass as shown in Brembilla et al. (2015a) and Page (2011). This second method to measure the efficiency is here defined with the name efficiencies of heat losses.

The current study aims to understand how the solar radiation and the geographic location influence the efficiency factors computed with the two methods before mentioned. In particular, this study intends to answer to the following Research Questions RQs:

a) Accuracy how would it be possible to consider the efficiency factors as a method to assess the performance of a space heating during both the design phase and for existing buildings?

b1) Predictions how would it be possible to predict the efficiency factors of a space heating located in cold climates for both methods?

b2) Relation how would it be possible to state whether the methods (common practice and efficiencies of heat losses) are related or not?

c) Paradoxes how would it be possible to consider the efficiency factor as a benchmark for the designing of the space heating by dissolving the paradoxes on this topic?

To study how the solar radiation and the geographic location affect the efficiency factors, a room model equipped with 1D model of hydronic floor heating system (validated in Brembilla et al. (2018)) was developed with the building energy simulation BES software IDA ICE vers. 4.7.1. The efficiency factor methods were assessed for Swedish localities to bring to light the relation between the efficiency factors and the solar radiation.

\section{Methodology}

The common practice method

The efficiency of the space heating is computed by assessing the ratio of the useful thermal energy released from the pipe loop upwards, $\mathrm{Q}_{\text {up }}$, over the thermal energy supplied to the space heating, $\mathrm{Q}_{\text {sup }}$. Both energies are shown in Fig. 1.

\section{The efficiencies of heat losses method}

The efficiency factors are computed by assessing the thermodynamic in/efficiencies of each building component such as, control, ceiling, window, and embedded surfaces. The thermodynamic in/efficiencies are computed by evaluating the heat losses of each component. The heat losses are a coupled problem between the heat emitted from the heat emitter and the heat lost through the building envelope. The efficiency factors are quantified with the ratio of the heat losses calculated with an ideal case over the heat losses of the real case.

The ideal case represents a space heating which uses the minor amount of thermal energy supplied for heating the indoor environment. This is because an ideal space heating uses "effectively" the thermal energy from free sources (e.g., solar radiation, electrical appliances, etc.). By doing that, an ideal space heating keeps the desired indoor temperature constant during the heating period. To achieve this goal, an ideal/theoretical local control adjusts the amount of heat supplied to the room control volume. Instead, the real cases have set a real control. The real control is unable to exploit "effectively" the free thermal energy from heat sources by allowing the indoor temperature to fluctuate.

Figure 2 shows a picture of how a local control of the space heating works. A sensor measures the room/operative temperature sending signals to the return valve. The return valve adjusts the amount of mass flow rate supplied, $\dot{\mathrm{m}}$, according to the control strategy adopted. The adjustment of $\dot{m}$ varies the heat supplied and as a result the heat emitted/released into the room. Both ideal and real cases use the same 
Fig. $1 \mathrm{Q}_{\text {sup }}$ and $\mathrm{Q}_{\text {up }}$ of the hydronic floor heating. a $\mathrm{Q}_{\text {sup }}$ to the floor heating. b $Q_{\text {up }}$ towards the floor

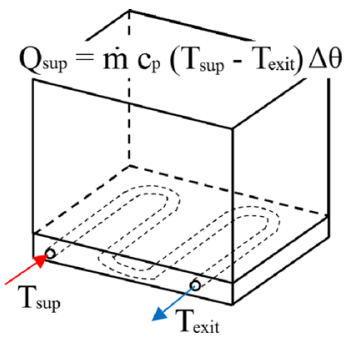

(a) Qsup to the floor heating

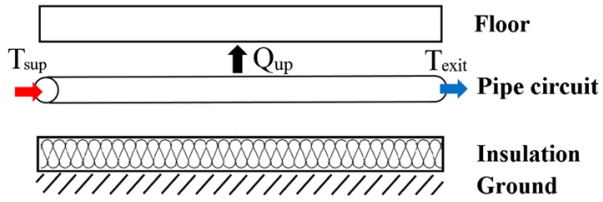

(b) Qup towards the floor equations for computing the thermal heat losses. The input values change from time to time (e.g., mass flow rate, supply temperature, etc) and the parameters remain constants (e.g., surface area, specific heat capacity, etc). Equation 1 accounts for the thermal energy supplied to the floor heating for both ideal and real cases.

$\mathrm{Q}_{\text {ctrl }, \text { ideal } / \text { real }}(\theta)=\sum_{i=1}^{n} \dot{\mathrm{m}}_{\mathrm{i}} \cdot \mathrm{c}_{\mathrm{fld}} \cdot\left(\mathrm{T}_{\text {sup }, \mathrm{i}}-\mathrm{T}_{\text {exit }, \mathrm{i}}\right) \cdot \Delta \theta_{\mathrm{i}}$

$c_{p}$ is the specific heat capacity of the heating medium, $\mathrm{T}_{\text {exit }}$ is the temperature exiting from the pipe circuit, $\mathrm{T}_{\text {sup }}$ is the supply temperature to the pipe circuit (outdoor temperature compensating), $\Delta \theta$ the time step, and $\mathrm{n}$ are the number of time steps. The ratio between the energy of the ideal case, $\mathrm{Q}_{\mathrm{ctrl}, \text { ideal }}$, and the energy of the real case, $\mathrm{Q}_{\mathrm{ctrl} \text {,real }}$, is named efficiency factor for control, $\eta_{\mathrm{ctrl}}$.

The other efficiency factors of the floor heating are related to the other heat losses as shown in Fig. 2.

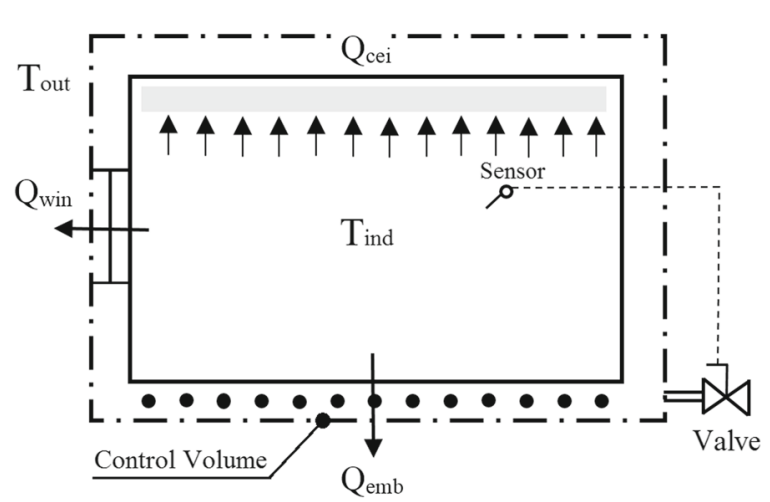

Fig. 2 Room control volume with heat losses of ceiling, window, and embedded surface
$\mathrm{Q}_{\text {str }}$ (which does not appear in Fig. 2) refers to the sum of heat losses adjacent to the ceiling, $\mathrm{Q}_{\text {cei }}$, where the indoor temperature is affected by over temperature, and the heat losses through the window, $\mathrm{Q}_{\text {win }}$, where the indoor temperature is influenced by the cold surface. Equation 2 calculates the heat losses for both ideal and real cases by assessing the heat losses from the indoor temperature, $\mathrm{T}_{\text {ind }}$, to the outside temperature $\mathrm{T}_{\text {out }}$.

$\mathrm{Q}_{\mathrm{j}, \text { ideal } / \text { real }}(\theta)=\sum_{\mathrm{j}=1}^{\mathrm{m}} \sum_{\mathrm{i}=1}^{\mathrm{n}} \mathrm{A}_{\mathrm{j}} \cdot \mathrm{U}_{\mathrm{j}, \mathrm{i}}(\theta) \cdot\left(\mathrm{T}_{\text {ind }, \mathrm{i}}(\theta)-\mathrm{T}_{\text {out }, \mathrm{i}}(\theta)\right) \cdot \Delta \theta_{\mathrm{i}}$

$\mathrm{U}_{\mathrm{j}}$ is the thermal transmittance coefficient of the $j$ th component and $\mathrm{A}_{\mathrm{j}}$ is its surface area.

Figure 3 shows the embedded heat losses of the hydronic floor heating system when the room is adjacent to the ground. Qemb refers to the sum of heat losses from the pipe loop downwards to the concrete slab named $\mathrm{Q}_{\mathrm{pl}}$, plus the heat losses from the insulating layer downwards to the ground defined as Qins. The embedded heat losses are calculated according to Eq. 3 when the slab faces outside, an unheated space and adjacent to the ground.

$\mathrm{Q}_{\mathrm{k}, \text { ideal } / \text { real }}(\theta)=\sum_{\mathrm{k}=1}^{\mathrm{m}} \sum_{\mathrm{i}=1}^{\mathrm{n}} \mathrm{A}_{\mathrm{k}} \cdot \frac{1}{\mathrm{R}_{\mathrm{k}, \mathrm{i}}} \cdot\left(\mathrm{T}_{\mathrm{k}, \mathrm{i}}(\theta)-\mathrm{T}_{\mathrm{k}+1, \mathrm{i}}(\theta)\right) \cdot \Delta \theta_{\mathrm{i}}$

$\mathrm{R}$ refers to the thermal resistance of the $k$ th component either the pipe loop or the insulating layer.

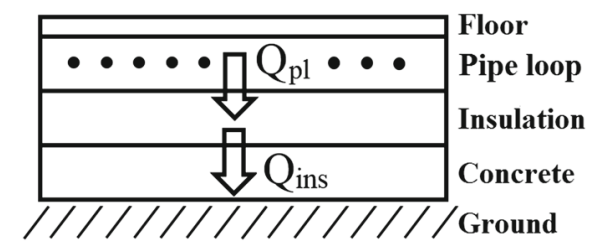

Fig. 3 Floor heating with pipe loop and insulation heat losses 
The efficiency factor for "stratification" $\eta_{\text {str }}$ is computed as the average value between the efficiency factors of heat losses of the ceiling and window as shown in Eq. 4.

$\eta_{\mathrm{str}}=\frac{\frac{\mathrm{Q}_{\text {cei,ideal }}}{\mathrm{Q}_{\text {cei,real }}}+\frac{\mathrm{Q}_{\text {win,ideal }}}{\mathrm{Q}_{\text {win,real }}}}{2}$

The efficiency factor of embedded surfaces $\eta_{\mathrm{emb}}$ is calculated as the average value between the efficiency factors of heat losses of pipe circuit and the insulating layer.

The determination of the total efficiency factor of the heated space, $\eta_{\text {tot }}$, can be calculated by Eq. 5 as reported in Olesen and De Carli (2011).

$\eta_{\mathrm{tot}}=\frac{1}{4-\left(\eta_{\mathrm{ctrl}}+\eta_{\mathrm{str}}+\eta_{\mathrm{emb}}\right)}$

where $\eta_{\mathrm{ctrl}}, \eta_{\mathrm{str}}, \eta_{\mathrm{emb}}$ have max values of 1 and min value of 0.4 . The latter coefficient was obtained by trial and error analysis.

Brief overview of the BES model

The simulation model consists of a room adjacent to other heated rooms in all directions except the wall facing the outside environment and the floor adjacent to the ground. Ideally, no heat passes through the adjacent conditioned rooms; thus, the three internal walls and the ceiling have the adiabatic boundary condition set. The building is oriented with the window facing South. Table 1 lists the thermal performance of the space heating and the characteristics of the heating system. Figure 4 illustrates the simulation model developed with IDA ICE vers. 4.7.1.

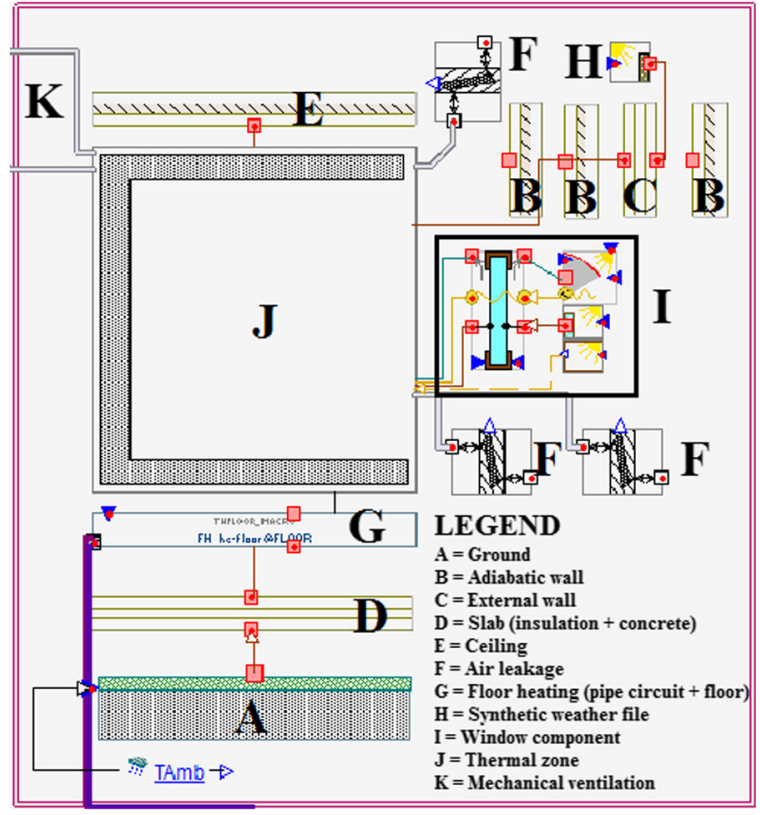

Fig. 4 Simulation model of the space heating developed with IDA ICE vers. 4.7.1

The ground model calculates the temperature of the virtual layer adjacent to the slab by applying the mean outdoor temperature at the steady-state and periodic thermal transmittance coefficients. Empirical calculations were made to assess the maximum thermal power of the hydronic floor heating $\left(69 \mathrm{Wm}^{-2}\right)$. Consequently, the achievable maximum mass flow rate of the heating medium was $0.083 \mathrm{~kg}^{-1}$, by assuming that the design flow temperature dropped along the pipe circuit of $5 \mathrm{~K}$. The mass flow rate was adjusted with thermostat ON/OFF control and the maximum electrical power of the circulating pump was $15 \mathrm{~W}$.
Table 1 Space heating thermal performance and characteristics of heating system addressed to the BES model

\begin{tabular}{llll}
\hline & Description & Value & Surface \\
\hline $\mathrm{U}_{\text {wall }}$ & Exterior wall & $0.15 \mathrm{Wm}^{-2} \mathrm{~K}^{-1}$ & $9.3 \mathrm{~m}^{2}$ \\
$\mathrm{U}_{\text {win }}$ & Window & $1.10 \mathrm{Wm}^{-2} \mathrm{~K}^{-1}$ & $2.7 \mathrm{~m}^{2}$ \\
$\mathrm{~g}_{\text {value }}$ & Solar heat gain coefficient & 0.33 & \\
$\mathrm{R}$ & Insulating layer & $6.25 \mathrm{~m}^{2} \mathrm{~K}^{1} \mathrm{~W}^{-1}$ & $25 \mathrm{~m}^{2}$ \\
$\mathrm{~L}$ & Pipe circuit length & & $82 \mathrm{~m}$ \\
$\varnothing$ & Pipe circuit inner diameter & & $0.017 \mathrm{~m}$ \\
$\dot{\mathrm{m}}$ & Design mass flow rate & $0.083 \mathrm{~kg}^{-1}$ & \\
$\mathrm{H}_{\mathrm{g}}$ & Ground steady-state coefficient & $6.4 \mathrm{WK}^{-1}$ & \\
$\mathrm{H}_{\mathrm{pe}}$ & Ground periodic coefficient & $3.1 \mathrm{WK}^{-1}$ & \\
$\dot{\mathrm{V}}$ & Mechanical ventilation supply flow rate & $10 \mathrm{ss}^{-1}$ & \\
$\dot{\mathrm{v}}$ & Air leakage of external surface & $0.2 \mathrm{ls}^{-1} \mathrm{~m}^{-2}$ & \\
\hline
\end{tabular}


The heating medium was stored in a tank (volume 3001 ) heated by an electrical resistor (power of $3 \mathrm{~kW}$ ). The electrical resistor provided the needed energy for fulfilling the heating medium requirements. The ventilation was supplied with a constant air volume system by providing a flow rate of $10 \mathrm{ls}^{-1}$.

To isolate the effects of the weather variables on the efficiency factors, the internal gains from occupancy, lighting, and electrical appliances were turned off during the simulations.

Synthetic weather file

The current paper uses climatic data taken from the synthetic weather file recorded in the International Weather Files for Energy Calculations 2.0 (IWEC2) described in Huang et al. (2014). The solar radiation, composed by direct and diffuse components, was predicted by using a regression analysis on data collected of outside dry-bulb temperature, relative humidity of air, wind speed and cloudiness factor as described in Zhang et al. (2002), Bre and Fachinotti (2016).

The recorded data of climatic variables have missing information which are predicted with several techniques, such as linear interpolation, Fourier series, etc. To tune the regression coefficients, Huang overcame this problem by using the measured data at regional level reported by Köppen-Geiger described in Kottek et al. (2006).

The synthetic climate file was reported in ASHRAE (2011) database and used it in IDA ICE as well as the geographic coordinates for latitude, longitude, and elevation of the site of each locality.

\section{Results and discussion}

Model's predictions of efficiency factors

Figure 5 illustrates the correlation between the total efficiency factor, $y$-left axis against the relative distance from North to South of five Swedish localities. The relative distance was calculated by means of ellipsoidal coordinates. The $y$-right axis presents the amount of heat gained from (direct + diffuse) solar radiation.

Figure 6 describes the relation between the total efficiency factor and the amount of heat gained from solar radiation into the room control volume. The total efficiency factor was computed with both methods

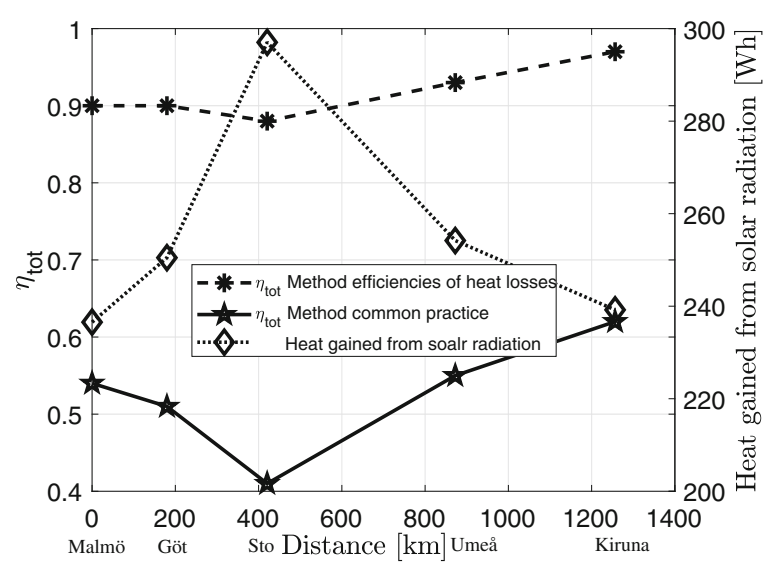

Fig. 5 Total Efficiency factor against the relative distance among localities computed with both methods

by collecting data for 13 Swedish localities. A linear regression model is applied to train the simulated data according to the ordinary least square method. The linear regression calculates an equation that minimizes the sum of squared residuals. The model is linear because of the linearity of the fitted curve coefficients. The model was tested assessing the determination coefficient $R^{2}$ which provides a measure of how well the data are replicated by the model's predictions as stated in Amasyali and El-Gohary (2018).

Figure 7 shows the total efficiency factor against the direct + diffuse solar radiation striking on the horizontal surface of the same chosen Swedish localities.

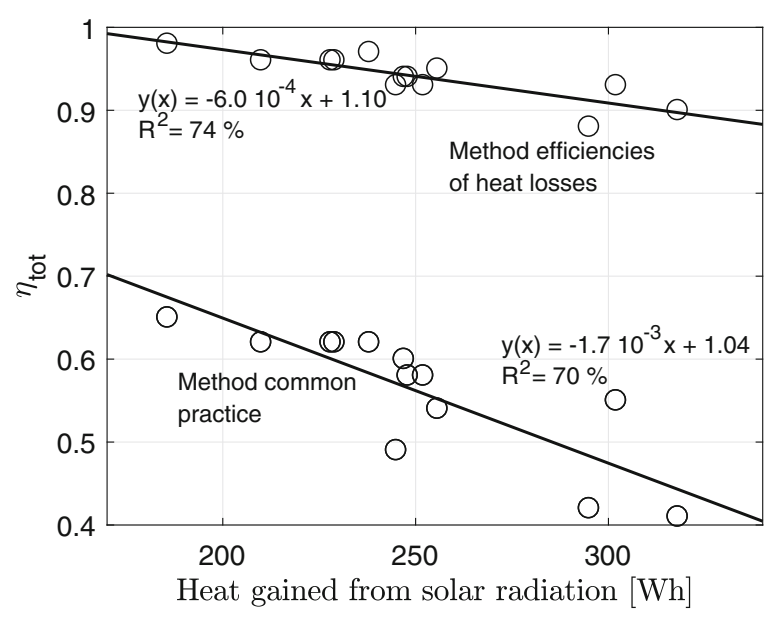

Fig. 6 Total efficiency factor against the heat gained from direct + diffuse solar radiation into the room control volume computed with both methods 


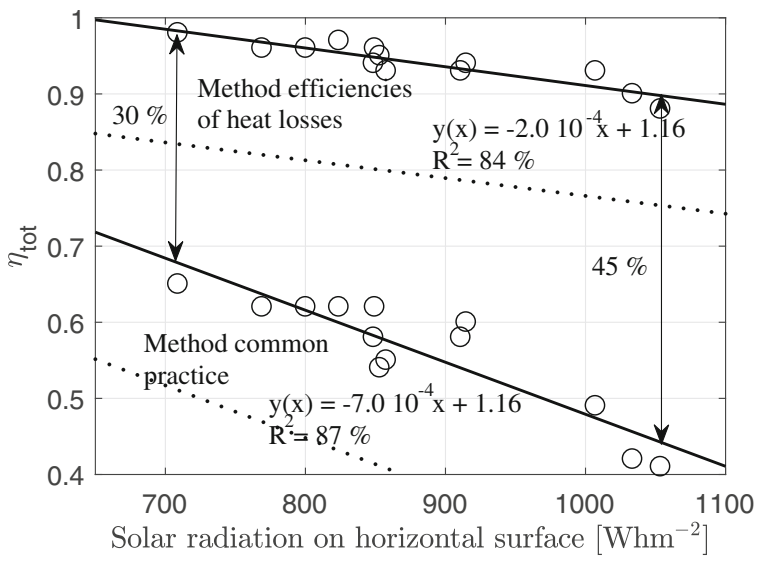

Fig. 7 Total efficiency factor against direct + diffuse solar radiation striking on horizontal surface computed with both methods

The same figure shows two additional functions, the dotted functions, which represent the efficiency curves that intercept the value of 1 .

\section{Discussion}

\section{Discussion on the efficiency factor methods}

The common practice for calculating the efficiency of the space heating can be misleading. At first glance, a researcher could think that this method calculates the efficiency of the floor heating without encompassing the thermal behavior of the room above the floor. In reality, this method computes the efficiency of the entire room control volume because both heat supplied and emitted upwards are regulated by the control strategy.

The efficiencies of heat losses method provides efficiency values for ceiling, window, and embedded surfaces close to 1 (higher than 0.99). This means that the energy lost through those building components is the same for both ideal and real cases. This fact is also confirmed by the Technical Standard EN 15316-2-1 (2007), which does not list the efficiency factors for ceiling and window in the case of hydronic floor heating being set. It is usefull to remind that the efficiency factors of the space heating studied are mainly influenced by the type of control used. ON/OFF control, case studied, allows the indoor temperature to fluctuate providing a "peaks" profile; whereas, other types of control, such as P and PI, would flatten the indoor temperature profile by providing higher efficiency factors.

The current study computes efficiency factors based on the ratio of energies for both methods proposed. Other studies, see Costa et al. (2006), account for the efficiency of air curtain as a ratio of heat transfer rates in steady-state condition. The energies computed in the current study are assessed with the BES software that encompasses the transient response of the building at temperature and load variations throughout the simulations. Therefore, it is reasonable to conclude that the efficiency factors measure the dynamic efficiency of the space heating.

\section{Discussion on the BES model and synthetic climate file}

Due to the relatively higher thermal inertia of the ground in comparison with the floor slab, the heat transfer rate exchanged between ground and floor slab can be split into two components: a steady state $\mathrm{H}_{\mathrm{g}}$, and a periodic part $\mathrm{H}_{\mathrm{pe}}$ (see Table 1). Both coefficients, $\mathrm{H}_{\mathrm{g}}$ and $\mathrm{H}_{\mathrm{pe}}$, depend on the room geometry and the ground thermal performance as described in detail in Hagentoft (2002).

The synthetic climate file used, IWEC2, is a tradeoff between the prediction's accuracy and the amount of available observations needed to predict the solar radiation. Fifty solar radiation models were revised in Menges et al. (2006). The authors concluded that the model able to predict most accurately the daily global radiation was developed in Ertekin and Yaldz (1999). The drawback of the latter model was in the amount of data needed (nine) for predicting the solar radiation. Therefore, the synthetic weather file developed in Huang et al. (2014), applied in the current study, represents a compromise between amount of data needed (five variables), complexity and elaboration time.

\section{Answer to $R Q$ a) Accuracy}

The research question (a) poses the problem whether the efficiency factors calculated with 1D model of hydronic floor heating could be applied both during the design phase and for the appraisal of existing space heating. The answer to such question is to seek in the accuracy of the numerical model used to compute the heat transfer rates/losses. 
A recent study, see Brembilla et al. (2018), validated the 1D model of hydronic floor heating (used in the current paper) developing an innovative validation methodology. The study applied the validation technique developed in Lomas (1991) (recently applied in Strachan et al. (2016)) at the model predictions obtained in a booth simulator. The booth simulator reduced the amount of disturbances (from outdoor and indoor climate) by affecting the thermal output of the floor heating (heat released upwards $\dot{\mathrm{q}}_{\text {up }}$ ) and the average floor surface temperature $\left(\bar{T}_{f}\right)$. The results showed the model predictions accuracy between 0.5 and $1.4 \%$ for $\overline{\mathrm{T}}_{\mathrm{f}}$ and between 2.2 and $6.2 \%$ for $\dot{\mathrm{q}}_{\mathrm{up}}$ when the pipe circuit has tube spacing of $0.30 \mathrm{~m}$. The model accuracy itself has larger plausibility bounds than the significant digits considered for estimating the efficiency factors $( \pm 1 \%)$. This means that the outcomes of 1D model of hydronic floor heating are suitable for predicting efficiency factors during the design phase of the space heating but not for the assessment of those factors for existing buildings.

Another question that arises is whether the number of time steps to estimate the predictions of 1D hydronic floor heating model has a beneficial impact (increased accuracy) on its outcomes. The increased accuracy of the model predictions could enable to use this model for the appraisal of the efficiency factors of existing space heating. IDA is a variable time step solver based on systems of differential algebraic equations as described in Balocco et al. (2015), Rohdin and Moshfegh (2007). As mentioned in Brembilla et al. (2018), by trying smaller time step, the accuracy of the model predictions remain unchanged for the significant digits considered in estimating the accuracy of $\dot{\mathrm{q}}_{\text {up }}$ and $\overline{\mathrm{T}}_{\mathrm{f}}$. The latter consideration confirms that the efficiency factors can be predicted exclusively during the design phase of the space heating.

It is worthwhile to mention that the type of integration method could decrease the accuracy of the efficiency factors. The current study integrates the heat losses by applying the trapezoidal rule. The trapezoidal rule calculates the area of each trapeze considering as trapeze basis the heat losses of following points and as trapeze height, the time step. Such rule is found to be less accurate than the mid-point and Simpson rule for functions, such as $x^{n}$ with $n \neq 1$ as shown in Eldén and Wittmeyer-Koch (1990), Ortiz and Popov (1985). By looking at generic heat losses in Fig. 8, the "local function" between following points

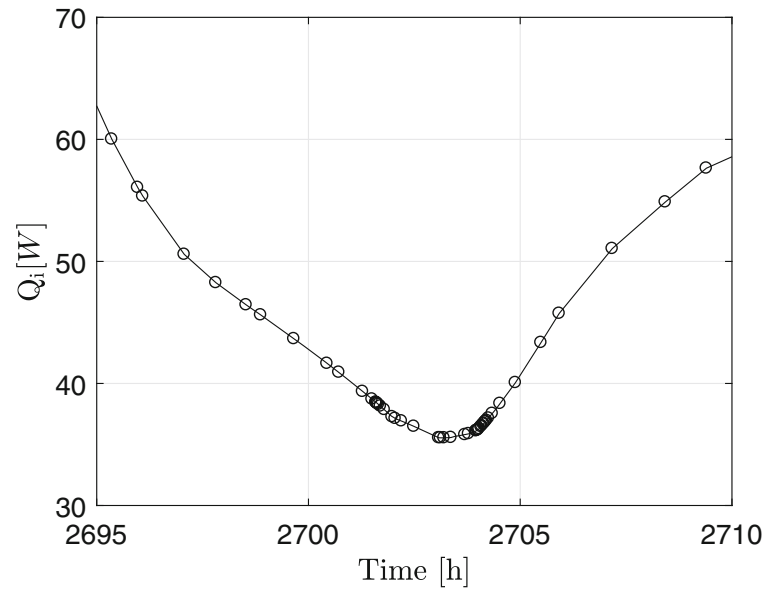

Fig. 8 Nodal points for a generic heat loss

is $x^{1}$, a linear function. The trapezoidal rule provides an exact integration for this function. Therefore, the integration method used does not degrade the accuracy of the efficiency factors.

\section{Answer to RQ b1) Predictions}

The total heat gained for Kiruna, Umeå and Stockholm seems correlated with the total efficiency factors in Fig. 5. The efficiency factors decrease of 7 and $4 \%$ (for the respective methods) from Kiruna to Umeå reporting an increase of heat gained of $6 \%$ between the localities. This is most likely due to the less disturbances caused by the heat gained from the solar radiation which makes less indoor temperature oscillations in Kiruna. Kiruna has an indoor temperature which is stable for a longer time than in Umeå and Stockholm. The latter fact is reflected in an increase of the total efficiency factor. The total efficiency factor for Stockholm is 41 and 88\%, 14/5 and 21/9 point percentages lower than Umeå and Kiruna, respectively. The total efficiency factor is at 51 and $90 \%$ and 54 90\% for Göteborg and Malmö although the two cities show a difference of heat gained from solar radiation of about $6 \%$.

From the latter figure, the efficiency factors do not have a clear correlation with the geographic location of localities.

To highlight more how the heat gained from solar radiation affects the efficiency factors, Fig. 6 shows this correlation for 13 Swedish localities. The locality with the highest efficiency is Abisko with 98 and 65\%. 
Stockholm is the lowest with 88 and $42 \%$. It is possible to notice a difference in 10 and 23 point percentage of efficiency between Abisko and Stockholm, by reporting a difference in heat gained of $37 \%$. A firstorder polynomial function is chosen for predicting the total efficiency factor. The latter choice is due to the fact that the efficiency changes of 10 and 23 point percentage against $135 \mathrm{Wh}$ of heat gained. The coefficient of $x$ is almost 0 as shown in Fig. 6. This means that the linear regression models proposed are almost flat.

The correlation between the solar radiation, which strikes on horizontal surface, and the total efficiency factor is depicted in Fig. 7. The regression analysis provides models with coefficients of determination of 84 and $87 \%$ by measuring a better fit in comparison with the case shown in Fig. 5 (74 and 70\%). The computed regression models are:

$$
\begin{aligned}
& y(x)=-2.0 \cdot 10^{-04} \mathrm{x}+1.16 \\
& y(x)=-7.0 \cdot 10^{-04} \mathrm{x}+1.16
\end{aligned}
$$

\section{Answer to $R Q$ b2) Relation}

Both results, shown in Fig. 7, have a similar trend by reporting differences between the curves in Eqs. 6 and 7 of about $30-45 \%$ evaluated at the boundary points of their validity range $\left(710-1060 \mathrm{Whm}^{-2}\right)$. The difference is because the two methods measure different efficiencies as addressed in the Introduction section. But, both methods are related because they are applied to the same domain. Simply put, both methods measure different efficiencies of the same space heating with applied same boundary conditions and input values. The relation between methods is further stressed by the fact that both fitting functions present a negative angular coefficient of the slope. The regression model in Eq. 7 presents the slope 3.5 times steeper than the curve in Eq. 6. The most notable common trend between curves is that the efficiency of space heating located in climates with lower solar radiation has higher efficiency factor than space heating located in climates with higher solar radiation. This is because cold climates provide less disturbances on the room temperature due to less amount of heat gain (from solar radiation) entering into the room control volume. Less disturbances on the indoor temperature result in less operations of the return valve for adjusting the mass flow rate supplied to the floor. Less valve operations produce an increase of the efficiency factors. Similar considerations "colder climates increase the efficiency" were also confirmed by the recent studies in Goubran et al. (2017). The latter study stated that air curtains located in Fabrikas (Alaska) provide about 30\% higher efficiency than the same air curtain located in Miami (Florida).

The fitting functions computed in Eqs. 6 and 7 present intercept values higher than 1. Ideally, the intercept has to be 1 when the solar radiation (located on the $x$-axis) has the value of 0 . Such ideal functions are plotted with dotted lines in Fig. 7. In principle, the angular coefficient of the slope can also change. The fact that the intercepts of the regression model are higher than 1 can be attributed to the disturbances on the heat losses caused from the wind, cloudiness factor, and geographical location.

\section{Answer to $R Q$ c) Paradoxes}

A misinterpretation of the results showed in Fig. 7 is that the best place to build buildings is in cold climates because it is possible to achieve higher efficiencies. This is the paradox in understanding how to interpret the efficiency factor. The correct interpretation is that cold climates "effectively" use the energy input into the system. The term "effectively" means that the percentage of useful energy used is higher in cold than in mild climates.

The second paradox in using the efficiency factor is to apply it as one value for designing the space heating of the entire country as made in EN 15316-2-1 (2007). One could think to have a threshold value of efficiency (e.g. 90/55\%) set equal for all locations considered. A single threshold value allows different configurations of the space heating. In practice, places located with low amount of solar radiation could have less thickness of insulation layer located underneath the pipe loop or larger windows, than space heating located in mild climates. This type of solution is in contrast with the basic principle of energy use. In fact, the modification of the space heating setup before mentioned would bring an even higher energy demand for space heating located in colder climates.

To synthesize, the efficiency factor method of the space heating has to be used as a performance indicator related to the outdoor environment conditions of each single locality. 


\section{Conclusion}

This work finds consistency and coherence in predicting the therml energy efficiency factors of a space heating equipped with hydronic floor heating located in cold climate. The initial hypothesis which stated that the efficiency factors vary according to the climate of each locality is fulfilled. The present work contributes to the field of efficiency factors of space heating by answering to the RQs:

a) Predictions of the efficiency factors are suitable to use during the design phase of the space heating. This is because the plausibly bounds of validated outcomes of 1D model of hydronic floor heating are higher than the significant digits considered for estimating the efficiency factors $( \pm 1 \%)$.

b1) The efficiency factors are correlated with the solar radiation striking on horizhontal surface. Two linear regression model fits the simulated data $\left(R^{2}=84\right.$ and $\left.87 \%\right)$ for predicting the total efficiency factor.

b2) Both methods for computing the efficiency factors, common practice and efficiencies of heat losses, are related because their predictions follow the same trend.

c) The efficiency factor method can be used as benchmark parameters to assess the performance of space heating related to the local outdoor climate. The latter remark on the efficiency factors dissolves the paradox on this topic that aimed to consider the efficiency factor as a benchmark indicator of the whole country.

\section{Outlook and future research}

The present work opens a further question: how would it be possible to compute the efficiency factors by avoiding the use of commercial BES software? The answer to such question may be found in Brembilla et al. (2015a, b, 2016).

\section{Nomenclature}

\section{Symbols}

$\begin{array}{lll}\mathrm{Q} & \text { Heat loss } & \mathrm{kWh} \\ \Delta & \text { Difference } & \\ \dot{\mathrm{m}} & \text { Mass flow rate } & \mathrm{kgs}^{-1} \\ \eta & \text { Efficiency } & \\ \theta & \text { Time } & \mathrm{s}\end{array}$

$\begin{array}{lll}\text { A } & \text { Area } & \mathrm{m}^{2} \\ \mathrm{c} & \text { Specific heat capacity } & \mathrm{Jkg}^{-1} \mathrm{~K}^{-1} \\ \mathrm{R} & \text { Thermal resistance } & \mathrm{m}^{2} \mathrm{~K}^{1} \mathrm{~W}^{-1} \\ \mathrm{~T} & \text { Temperature } & \mathrm{K} \\ \mathrm{U} & \text { Thermal transmittance } & \mathrm{Wm}^{-2} \mathrm{~K}^{-1} \\ \text { Subscripts } & \\ \text { cei } & \text { Ceiling } & \\ \text { win } & \text { Window } \\ \text { ctrl } & \text { Control } \\ \text { emb } & \text { Embedded } \\ \text { exit } & \text { Exiting } \\ \text { fld } & \text { Fluid } \\ \text { ind } & \text { Indoor } \\ \text { str } & \text { Stratification } \\ \text { tot } & \text { Total } \\ \text { up } & \text { Upwards } \\ \text { out } & \text { Outdoor } \\ \text { sup } & \text { Supply } \\ \text { Acronyms } & \\ \text { BES } & \text { Building Energy Simulation } \\ \text { ICE } & \text { Indoor Climate and Energy } \\ \text { IDA } & \text { Implicit Differential Algebraic solver } \\ \text { IWEC2 } & \text { International Weather for Energy } \\ & \text { Calculations 2 } \\ \text { RQ } & \text { Research Question } \\ \end{array}$

Acknowledgments Christian Brembilla wish to express his gratitude to Annika Bindler for correcting English language mistakes in the text. The manuscript, at its first submission, had reported the following comments of four out of five anonymous reviewers: 1) The manuscript is well structured, easy to read and follow. The subject is interesting and the manuscript gives increased knowledge to the field of space heating, 3) The paper is well written and clear,... 4) ...positive evaluation of the article as a whole 5) To my understanding the work is done in a very good quality, I trust the results.

Open Access This article is distributed under the terms of the Creative Commons Attribution 4.0 International License (http:// creativecommons.org/licenses/by/4.0/), which permits unrestricted use, distribution, and reproduction in any medium, provided you give appropriate credit to the original author(s) and the source, provide a link to the Creative Commons license, and indicate if changes were made.

\section{References}

Abdullah, Y., \& Ali, G. (2009). Energy and exergy analyses of space heating in buildings. Applied Energy, 86(10), 19391948 . 
Amasyali, K., \& El-Gohary, N.M. (2018). A review of datadriven building energy consumption prediction studies. Renewable and Sustainable Energy Reviews, 81, 11921205.

Asan, H. (2006). Numerical computation of time lags and decrement factors for different building materials. Building and Environment, 41(5), 615-620.

ASHRAE. (2011). American society of heating refrigerating and air conditioning engineers. Atlanta: Weather Files.

Balocco, C., Petrone, G., Cammarata, G., Vitali, P., Albertini, R., Pasquarella, C. (2015). Experimental and numerical investigation on airflow and climate in a real operating theatre under effective use conditions. International Journal of Ventilation, 13(4), 351-368.

Bre, F., \& Fachinotti, V.D. (2016). Generation of typical meteorological years for the Argentine Littoral Region. Energy and Buildings, 129, 432-444.

Brembilla, C. (2016). One dimensional model of transient heat conduction through multilayer walls/slabs : the functionality of insulation and brick materials in terms of decrement factor and time lag, in Licentiate Dissertation, Department of Applied Physics and Electronics, Umeå University.

Brembilla, C., Lacoursiere, C., Soleimani-Mohseni, M., Olofsson, T. (2015a). Investigations of thermal parameters addressed to a building simulation model. In: Proceeding of the 14th International Conference of the International Building Performance Simulation Association IBPSA, Hyderabad, India, pp. 2741-2748.

Brembilla, C., Soleimani-Mohseni, M., Olofsson, T. (2015b). Transient model of a panel radiator. In: Proceedings of the 14th International Building Performance Simulation Association IBPSA, Hyderabad, India, pp. 2749-2756.

Brembilla, C., Vuolle, M., Östin, R., Olofsson, T. (2017). Practical support for evaluating efficiency factors of a space heating system in cold climates. Energy Efficiency, 10(5), 1253-1267.

Brembilla, C., Östin, R., Olofsson, T. (2018). Predictions' robustness of one-dimensional model of hydronic floor heating: novel validation methodology using a thermostatic booth simulator and uncertainty analysis. Journal of Building Physics, 41(5), 418-444.

Costa, J., Oliveira, L., Silva, M. (2006). Energy savings by aerodynamic sealing with a downward-blowing plane air curtain a numerical approach. Energy and Buildings, 38(10), 11821193.

Dinçer, I., \& Rosen, M. (2010). 6. Thermal energy storage, System and applications, 2nd edn., (pp. 237-238). New York: Wiley.

Eldén, L., \& Wittmeyer-Koch, L. (1990). 2. Numerical analysis, An introduction, 6th edn., (pp. 78-79). New York: Mc Graw Hill.

Emmel, M.G., Abadie, M.O., Mendes, N. (2007). New external convective heat transfer coefficient correlations for isolated low-rise buildings. Energy and Buildings, 39(3), 335-342.

EN 15316-2-1 (2007). Heating systems in buildings-method for calculation of system energy requirements and system efficiencies - Part 2-1.
Ertekin, C., \& Yaldz, O. (1999). Estimation of monthly average daily global radiation on horizontal surface for Antalya (Turkey). Renewable Energy, 17(1), 95-102.

Goubran, S., Qi, D., Wang, L.L. (2017). Assessing dynamic efficiency of air curtain in reducing whole building annual energy usage. Building Simulation, 10(4), 497-507.

Hagentoft, C.-E. (2002). Steady-state heat loss for an edgeinsulated slab: Part I. Building and Environment, 37(1), 19-25.

Huang, Y., Fenxian, S., Seo, D., Krarti, M. (2014). Development of 3012 IWEC2 weather files for international locations (RP-1477). ASHRAE Transactions, 120(1), 340-355.

Kottek, M., Grieser, J., Beck, C., Rudolf, B., Rubel, F. (2006). World Map of the Köppen-Geiger climate classification updated. Meteorologische Zeitschrift, 15(3), 259-263.

Lomas, K. (1991). Dynamic thermal simulation models of buildings: new method for empirical validation. Building Services Engineering Research and Technology, 12(1), 2537.

Loveday, D., \& Taki, A. (1996). Convective heat transfer coefficients at a plane surface on a full-scale building facade. International Journal of Heat and Mass Transfer, 39(8), 1729-1742.

Menges, H.O., Ertekin, C., Sonmete, M.H. (2006). Evaluation of global solar radiation models for Konya, Turkey. Energy Conversion and Management, 147(18-19), 3149-3173.

Olesen, B.W., \& De Carli, M. (2011). Calculation of the yearly energy performance of heating systems based on the european building energy directive and related CEN standards. Energy and Buildings, 43(5), 1040-1050.

Ortiz, M., \& Popov, E.P. (1985). Accuracy and stability of integration algorithms for elastoplastic constitutive relations. International Journal for Numerical Methods in Engineering, 21(9), 1561-1576.

Page, A.W. (2011). The role of thermal mass in the sustainable design of australian housing. Mauerwerk, 15(3), 179-185.

Paulescu, M., Tulcan-Paulescu, E., Stefu, N. (2011). A temperature-based model for global solar irradiance and its application to estimate daily irradiation values. International Journal of Energy Research, 35, 520-529, 5.

Rohdin, P., \& Moshfegh, B. (2007). Variable air volume-flow systems-a possible way to reduce energy use in the Swedish dairy industry. International Journal of Ventilation, 5(4), 381-392.

Rosen, M.A. (2002). Clarifying thermodynamic efficiencies and losses via exergy. Exergy, An International Journal, 2(1), 3-5.

Shao, J., Liu, J., Zhao, J., Zhang, W., Fu, Z., Zhu, Q. (2010). Field measurement of the convective heat transfer coefficient on vertical external building surfaces using naphthalene sublimation method. Journal of Building Physics, 33(4), 307-326.

Strachan, P., Svehla, K., Heusler, I., Kersken, M. (2016). Whole model empirical validation on a full-scale building. Journal of Building Performance Simulation, 9(4), 331-350.

Zhang, Q., Huang, J., Lang, S. (2002). Development of typical year weather data for Chinese locations. ASHRAE Transactions, 108(2), 1063-1075. 